\title{
GROUPS WITH MANY REWRITABLE PRODUCTS
}

\author{
MARIO CURZIO, PATRIZIA LONGOBARDI, \\ MERCEDE MAJ, AND AKBAR RHEMTULLA
}

(Communicated by Warren J. Wong)

\begin{abstract}
For any integer $n \geq 2$, denote by $R_{n}$ the class of groups $G$ in which every infinite subset $X$ contains $n$ elements $x_{1}, \ldots, x_{n}$ such that the product $x_{1} \cdots x_{n}=x_{\sigma(1)} \cdots x_{\sigma(n)}$ for some permutation $\sigma \neq 1$. The case $n=2$ was studied by B. H. Neumann who proved that $R_{2}$ is precisely the class of centre-by-finite groups. Here we show that $G \in R_{n}$ for some $n$ if and only if $G$ contains an $F C$-subgroup $F$ of finite index such that the exponent of $F / Z(F)$ is finite, where $Z(F)$ denotes the centre of $F$.
\end{abstract}

\section{INTRODUCTION}

B. H. Neumann proved in [8] that a group is centre-by-finite if and only if every infinite subset contains a pair of elements that commute. Extensions of problems of this type are to be found in [7] and [6]. The notion of commutativity was extended to rewritable products in [4] with a complete description obtained in [5]. Detailed study of rewritable groups may be found in papers by R. Blyth [1] and [2]. Following earlier authors, we call $G$ a $P_{n}$-group if given any sequence $x_{1}, \ldots, x_{n}$ of $n$ elements in $G$, their product $x_{1} \cdots x_{n}=x_{\sigma(1)} \cdots x_{\sigma(n)}$ for some permutation $\sigma \neq 1$. A group $G$ is called a $Q_{n}$ group if given any set $\left\{x_{1}, \ldots, x_{n}\right\}$ of $n$ elements of $G, x_{\sigma(1)} \cdots x_{\sigma(n)}=x_{\phi(1)} \cdots x_{\phi(n)}$ for some permutations $\sigma \neq \phi$. Clearly $P_{n} \subseteq Q_{n}$ and Blyth [1] has shown that $\bigcup_{n} P_{n}=\bigcup_{n} Q_{n}$.

Call $G$ an $R_{n}$ group if every infinite subset $X$ of $G$ contains a subset $\left\{x_{1}, \ldots, x_{n}\right\}$ of $n$ elements such that $x_{1} \cdots x_{n}=x_{\sigma(1)} \cdots x_{\sigma(n)}$ for some permutation $\sigma \neq 1$. Clearly $Q_{n} \subseteq R_{n}$ for every $n$. The relation of $R_{n}$ to $Q_{n}$ is rather like that of centre-by-finite groups to abelian groups. Our main result is the following.

Theorem A. A group $G$ is an $R_{n}$ group for some integer $n$ if and only if $G$ has a normal subgroup $F$ such that $G / F$ is finite, $F$ is an $F C$-group and the exponent of $F / Z(F)$ is finite.

Received by the editors November 7, 1990 and, in revised form, January 21, 1991.

1980 Mathematics Subject Classification (1985 Revision). Primary 20F24, 20 E34. 
Recall that a group $G$ is an $F C$-group if every element of $G$ has only finitely many conjugates in $G$. For results on $F C$-groups we refer the reader to [9].

\section{Proofs}

Groups $G$ in which, given any infinite sequence $x_{1}, x_{2}, \ldots$ of elements in $G$, there exist some $m \in \mathbb{N}$ and permutations $\sigma \neq \phi$ in $S_{m}$ such that

$$
x_{\sigma(1)} \cdots x_{\sigma(m)}=x_{\phi(1)} \cdots x_{\phi(m)}
$$

were studied in [3]. The class $Q_{\infty}$ of all such groups was shown to be precisely the class of $F C$-by-finite groups. Our first lemma makes use of this.

Lemma 1. If $G \in R_{n}$ for some $n$ then $G$ is $F C$-by-finite.

Proof. Let $G \in R_{n}$ and let $x_{1}, x_{2}, \ldots$ be any infinite sequence. Let $X=$ $\left\{x_{i} ; i=1,2, \ldots\right\}$. It contains $n$ elements, say $x_{\lambda_{1}}, \ldots, x_{\lambda_{n}}$ such that their product is rewritable. Let $\lambda=\max \left\{\lambda_{i} ; i=1, \ldots, n\right\}$. Consider the sequence $x_{1}, \ldots, x_{\lambda}$. Reorder the sequence as $y_{1}, \ldots, y_{\lambda}$ where $y_{i}=x_{\lambda_{i}}, i=1, \ldots, n$ and the rest of the $y_{i}$ 's are the rest of $x_{i}$ 's in some order.

Now $y_{1} \cdots y_{n}=y_{\sigma(1)} \cdots y_{\sigma(n)}$ for some $\sigma \neq 1$. Hence $y_{1} \cdots y_{n} y_{n+1} \cdots y_{\lambda}=$ $y_{\sigma(1)} \cdots y_{\sigma(n)} y_{n+1} \cdots y_{\lambda}$ and so $G \in Q_{\infty}$. Thus by Proposition 2 of [3], $G$ is $F C$-by-finite.

Lemma 2. Let $F$ be a normal subgroup of a group $G$ such that $G / F$ is finite of exponent $e, G / Z(F)$ is of finite exponent $e^{\prime}$ and $F$ is an FC-group. Then $G \in R_{e+e^{\prime}}$.

Proof. Since every finite group is in $R_{n}$ for every $n \geq 2$, we may assume that $G$ is not finite. Let $X$ be any infinite set in $G$. Then $|F g \cap X|=\infty$ for some $g \in G$. Thus we may as well assume that $X \subseteq F g$. Pick any $x_{1}, \ldots, x_{e}$ from $X$. Their product $x_{1} \cdots x_{e}=f$ lies in $F$. Let $X^{\prime}=X \backslash\left\{x_{1}, \ldots, x_{e}\right\}$. Since $F$ is an $F C$-group and $f \in F$, the centralizer $C$ of $\left\langle f^{G}\right\rangle$ in $F$ is of finite index in $G$; also $C \supseteq Z(F)$. Now $\left|X^{\prime} \cap C h\right|=\infty$ for some $h$ in $G$ so pick any $e^{\prime}$ elements $c_{1} h, \ldots, c_{e^{\prime}} h$ from $X^{\prime} \cap C h$. Then

$$
f\left(c_{1} h\right) \cdots\left(c_{e^{\prime}} h\right)=\left(c_{1} h\right) \cdots\left(c_{e^{\prime}} h\right) f^{h^{h^{\prime}}}=\left(c_{1} h\right) \cdots\left(c_{e^{\prime}} h\right) f
$$

for $h^{e^{\prime}} \in Z(F)$. Since $f$ is a product of $e$ elements of $X, G \in R_{e+e^{\prime}}$.

The above lemma provides the easy half of the proof of Theorem A. For the other half we need the following preliminary results.

Lemma 3. Let $G$ be an $F C$ group that is also nilpotent of class two. Then $G \in R_{n}$ for some $n \geq 2$ implies $G / Z(G)$ has finite exponent.

Proof. Suppose not. Then there exist $a_{1}, b_{1}$ in $G$ such that $\left[b_{1}^{i}, a_{1}\right]=c_{1}^{i} \neq 1$ for all $i=1, \ldots, n$. Clearly if we set $\bar{G}=G /\left\langle c_{1}\right\rangle$, the exponent of the group $\bar{G} / Z(\bar{G})$ is not finite, for $G$ is an $F C$-group so that $\left\langle c_{1}\right\rangle$ is finite. Next choose $b_{2}, a_{2}$ in $C_{G}\left\langle a_{1}, b_{1}\right\rangle$ such that $\left[b_{2}^{i}, a_{2}\right]=c_{2}^{i} \notin\left\langle c_{1}\right\rangle$ for all $i=1, \ldots, n$. This is possible since $C_{G}\left\langle a_{1}, b_{1}\right\rangle$ is of finite index in $G$.

Continue; at step $k$, pick $b_{k}, a_{k}$ in $C_{G}\left\langle a_{1}, \ldots, a_{k-1}, b_{1}, \ldots, b_{k-1}\right\rangle$ such that $\left[b_{k}^{i}, a_{k}\right]=c_{k}^{i}$ does not lie in $\left\langle c_{1}, \ldots, c_{k-1}\right\rangle$ for all $1 \leq i \leq n$. Now put $x_{0}=b_{1}$ and $x_{k}=a_{1} \ldots a_{k} b_{k+1}$ for $k>0$. Take $X=\left\{x_{i} ; i \geq 0\right\}$. We claim that if $\lambda_{1}, \ldots, \lambda_{n}$ are any $n$ distinct nonnegative integers, then $x_{\lambda_{1}} \cdots x_{\lambda_{n}}=$ $x_{\lambda_{\sigma(1)}} \cdots x_{\lambda_{\sigma(n)}}$ implies $\sigma=1$. 
For each $\lambda_{j}$ let $r\left(\lambda_{j}\right)$ denote the number of $x_{\lambda_{i}}$ 's with $\lambda_{i}>\lambda_{j}$ appearing to the right of $x_{\lambda_{j}}$ in the expression $x_{\lambda_{1}} \cdots x_{\lambda_{n}}$. Let $s\left(\lambda_{j}\right)$ denote the number of $x_{\lambda_{i}}$ 's with $\lambda_{i}>\lambda_{j}$ appearing to the right of $x_{\lambda_{j}}$ in the expression $x_{\lambda_{\sigma(1)}} \cdots x_{\lambda_{\sigma(n)}}$. Let $\lambda_{j}$ be the largest integer such that $r=r\left(\lambda_{j}\right) \neq s\left(\lambda_{j}\right)=s$. Then modulo $\left\langle c_{1}, \ldots, c_{\lambda_{j}}\right\rangle$,

where $k=\lambda_{j}+1$.

$$
\left(x_{\lambda_{1}} \ldots x_{\lambda_{n}}\right)\left(x_{\lambda_{\sigma(1)}} \ldots x_{\lambda_{\sigma(n)}}\right)^{-1} \equiv b_{k} a_{k}^{r} a_{k}^{-s} b_{k}^{-1} a_{k}^{s-r} \equiv c_{k}^{r-s},
$$

Since $1 \leq|s-r|<n, c_{k}^{s-r} \notin\left\langle c_{1}, \ldots, c_{\lambda_{j}}\right\rangle$ and hence $x_{\lambda_{1}} \cdots x_{\lambda_{n}}=x_{\lambda_{\sigma(1)}} \cdots x_{\lambda_{\sigma(n)}}$ only if $r\left(\lambda_{j}\right)=s\left(\lambda_{j}\right)$ for all $j$. But this implies that $\sigma=1$ and thus completes the proof.

Lemma 4. Let $G$ be an FC-group such that the exponent of $G / \zeta_{2}(G)$ is finite. If $G \in R_{n}$ for some $n \geq 2$, then the exponent of $G / \zeta_{1}(G)$ is finite. $\quad\left(\zeta_{i}(G)\right.$ denotes the ith centre of $G)$.

Proof. Write $Z_{i}$ for $\zeta_{i}(G)$. By Lemma 3, the exponent of $Z_{2} / \zeta_{1}\left(Z_{2}\right)$ is finite. Put $A=\zeta_{1}\left(Z_{2}\right)$.

It will suffice to show that the exponent of $A / Z_{1}$ is finite. Let $e$ be the exponent of $G / Z_{2}$ and suppose there is $a \in A$ such that $a^{e} \notin Z_{1}$. Then $\left[a^{e}, b\right] \neq 1$ for some $b \in G$. But $\left[a^{e}, b\right]=[a, b]^{e}=\left[a, b^{e}\right] \in\left[A, Z_{2}\right]=1$, a contradiction.

Proof of Theorem A. Let $G \in R_{n}$ for some $n \geq 2$. By Lemma 1, the $F C$-centre $F$ of $G$ is of finite index in $G$. Because of Lemma 2, we only need to show that $F / Z(F)$ has finite exponent to complete the proof. If $F$ is finite, there is nothing to prove, so we may assume $|F|=\infty$. If $F / \zeta_{2}(F)$ has finite exponent, then Lemma 4 applies and we are done.

If $F / \zeta_{2}(F)$ is not of finite exponent then consider the group $\bar{F}=F / Z(F)$ and get a contradiction by showing that the exponent of $\bar{F} / Z(\bar{F})$ is finite. The purpose of considering $F / Z(F)$ is that it is residually finite (see Theorem 1.9 of [9]). So we assume that $F$ is a residually finite $F C$-group in the class $R_{n}$ for some $n \geq 2$ and that the exponent of $F / Z(F)$ is not finite. Then there exist $a_{1}, b_{1}$ in $F$ such that $\left[a_{1}^{i}, b_{1}\right] \neq 1$ for all $i=1,2, \ldots, n$. Let $A_{1}=\left\langle\left[a_{1}, b_{1}\right]^{F}\right\rangle$. Note that $A_{1}$ is finite. Let $C_{1}=C_{F}\left(\left\langle a_{1}, b_{1}\right\rangle^{F}\right)$ so that $C_{1}$ is of finite index in $F$ and there exists $N_{1} \triangleleft G$ such that $N_{1} \leq C_{1}, N_{1} \cap$ $A_{1}=1$ and $F / N_{1}$ is finite. Note that $N_{1} / Z\left(N_{1}\right)$ is not of finite exponent for $F=\left\langle N_{1}, Y_{1}\right\rangle$ for some finite set $Y_{1}$ and $C_{F}\left(Y_{1}\right)$ has finite index so that $C_{F}\left(Y_{1}\right) \cap Z\left(N_{1}\right) \leq Z(F)$ is of finite index in $Z\left(N_{1}\right)$ and hence $F / Z(F)$ would have finite exponent.

Next choose $a_{2}, b_{2}$ in $N_{1}$ such that $\left[a_{2}^{i}, b_{2}\right] \neq 1$ for all $0<i \leq n$. Obtain subgroup $N_{2} \triangleleft F$ such that $N_{2} \leq N_{1} \cap C_{F}\left(\left\langle a_{2}, b_{2}\right\rangle^{F}\right), N_{2} \cap\left\langle\left[a_{2}, b_{2}\right]^{F}\right\rangle=1$, and $F / N_{2}$ is finite. This is possible and $N_{2}$ is obtained in a similar way to $N_{1}$. Also note that the exponent of $N_{2} / Z\left(N_{2}\right)$ is not finite.

Continue the above process to get the sequence $a_{3}, b_{3}, a_{4}, b_{4} \cdots$. Now put $x_{0}=b_{1}, x_{1}=a_{1} b_{2}, \ldots, x_{k}=a_{1} \cdots a_{k} b_{k+1}, \ldots$ and let $X=\left\{x_{i}, i \geq 0\right\}$. We claim that if $\lambda_{1}, \ldots, \lambda_{n}$ are any $n$ distinct nonnegative integers and

$$
x_{\lambda_{1}} \cdots x_{\lambda_{n}}=x_{\lambda_{\sigma(1)}} \cdots x_{\lambda_{\sigma(n)}}
$$

then $\sigma=1$. This is done by using an argument similar to that in Lemma 3 . This completes the proof. 


\section{REFERENCES}

1. R. D. Blyth, Rewriting products of group elements. I, J. Algebra 116 (1988), 506-521.

2. __ Rewriting products of group elements. II, J. Algebra 118 (1988), 249-259.

3. R. D. Blyth and A. H. Rhemtulla, Rewritable products in FC-by-finite groups, Canad. J. Math. 41 (1989), 369-384.

4. M. Curzio, P. Longobardi, and M. Maj, Su di un problema combinatorio in teoria dei gruppi, Atti. Accad. Naz. Lincei Rend. Cl. Sci. Fis. Mat. Natur. 8 (1983), 136-142.

5. M. Curzio, P. Longobardi, M. Maj, and D. J. S. Robinson, A permutational property of groups, Arch. Math. (Basel) 44 (1985), 385-389.

6. J. R. J. Groves, A conjecture of Lennox and Wiegold concerning supersoluble groups, J. Austral. Math. Soc. 35 (1983), 218-220.

7. J. C. Lennox and J. Wiegold, Extensions of a problem of Paul Erdös on groups, J. Austral. Math. Soc. 31 (1981), 459-463.

8. B. H. Neumann, A problem of Paul Erdös on groups, J. Austral. Math. Soc. 21 (1976), 467-472.

9. M. J. Tomkinson, FC-groups, Res. Notes in Math., no. 96, Pitman, London, 1984.

(M. Curzio, P. Longobardi, and M. Maj) Dipartimento di Matematica e Applicazioni, UniVersitá degli studi di Napoli, via Mezzocannone 8, 80134 Naples, Italy

(A. Rhemtulla) Department of Mathematics, University of Alberta, Edmonton, Canada T6G 2G1 